\title{
Synapses in the Fly Motion-Vision Pathway: \\ Evidence for a Broad Range of Signal Amplitudes and Dynamics
}

Ulrich Beckers, Martin Egelhaaf and Rafael Kurtz

J Neurophysiol 97:2032-2041, 2007. First published Jan 10, 2007; doi:10.1152/jn.01116.2006

You might find this additional information useful...

This article cites 33 articles, 15 of which you can access free at:

http://jn.physiology.org/cgi/content/full/97/3/2032\#BIBL

Updated information and services including high-resolution figures, can be found at:

http://jn.physiology.org/cgi/content/full/97/3/2032

Additional material and information about Journal of Neurophysiology can be found at: http://www.the-aps.org/publications/jn

This information is current as of March 13, 2007. 


\title{
Synapses in the Fly Motion-Vision Pathway: Evidence for a Broad Range of Signal Amplitudes and Dynamics
}

\author{
Ulrich Beckers, Martin Egelhaaf, and Rafael Kurtz \\ Department of Neurobiology, Bielefeld University, Bielefeld, Germany
}

Submitted 20 October 2006; accepted in final form 9 January 2007

Beckers U, Egelhaaf M, Kurtz R. Synapses in the fly motion-vision pathway: evidence for a broad range of signal amplitudes and dynamics. J Neurophysiol 97: 2032-2041, 2007. First published January 10, 2007; doi:10.1152/jn.01116.2006. Synapses are generally considered to operate efficiently only when their signaling range matches the spectrum of prevailing presynaptic signals in terms of both amplitudes and dynamics. However, the prerequisites for optimally matching the signaling ranges may differ between spikemediated and graded synaptic transmission. This poses a problem for synapses that convey both graded and spike signals at the same time. We addressed this issue by tracing transmission systematically in vivo in the blowfly's visual-motion pathway by recording from single neurons that receive mixed potential signals consisting of rather slow graded fluctuations superimposed with highly variable spikes from a small number of presynaptic elements. Both pre- and postsynaptic neurons were previously shown to represent preferred-direction motion velocity reliably and linearly at low fluctuation frequencies. To selectively assess the performance of individual synapses and to precisely control presynaptic signals, we voltage clamped one of the presynaptic neurons. Results showed that synapses can effectively convey signals over a much larger amplitude and frequency range than is normally used during graded transmission of visual signals. An explanation for this unexpected finding might lie in the transmission of the spike component that reaches larger amplitudes and contains higher frequencies than graded signals.

\section{N T R O D U C T I O N}

Synaptic transmission and signal processing are key features shaping the computational properties of neuronal networks. Whereas signal transfer between spiking neurons needs to distinguish only between spikes and noise, at synapses conveying graded potentials, a low signal-to-noise ratio is crucial to ensure a sufficiently large signaling capacity. A high gain in synaptic transmission improves graded signaling in the presence of noise, but limits the synapses' working range, which ideally should match the range of naturally occurring signals (Laughlin 1981).

Synaptic transmission between spiking neurons is already well documented (e.g., Redman 1990; Wadiche and Jahr 2001) and recent studies also addressed graded transmission (Field and Rieke 2002; Simmons 2002; Simmons and de Ruyter van Stevenick 2005). We investigated the transmission of signals consisting of both graded potentials and spikes of variable amplitude at synapses in the blowfly's visual system. At these mixed potential synapses, the optimal matching of synaptic signaling to the range of presynaptic signals seems problematic: On the one hand, the synapses have to capture the large

Address for reprint requests and other correspondence: U. Beckers, University Bielefeld, Department of Neurobiology, Postfach 1001 31, 33501 Bielefeld, Germany (E-mail: ubeckers1@uni-bielefeld.de). amplitude range covered by spikes and, in purely spike-mediated transmission, the signal-to-noise ratio may be enhanced by nonlinearities accentuating large and fast presynaptic signals at the cost of small and slow ones. On the other hand, small and slow signals need to be transmitted in a graded fashion, and the large amplitude range required for spike-mediated transmission will inevitably limit the synapse's resolution in the low-amplitude regime. This is a general problem at mixed potential synapses that convey both graded and spike-mediated signals. Such synapses have been known for a long time in invertebrate systems, for example, in leech segmental ganglia (e.g., Arbas and Calabrese 1987), crustacean stomatogastric ganglia (e.g., Graubard et al. 1980), or the insect visual system (e.g., Hengstenberg 1977; Juusola et al. 1995; Simmons 1982)—the latter serving as a model system in the present study. However, even in several prominent areas of vertebrate nervous systems in which synaptic transmission has long been regarded as purely spike mediated, it was recently shown that graded membrane potential components may invade distant presynaptic arbors (for review see Marder 2006). In such a case, the graded membrane potential component modulates transmitter release by either changing spike waveform (Alle and Geiger 2006; Shu et al. 2006) or by acting directly on the $\mathrm{Ca}^{2+}$-dependent exocytosis process (Awatramani et al. 2005).

The synapses investigated in the present study connect part of the blowfly's vertical system (VS) with the V1 cell (Kurtz et al. 2001). The VS cells consist of ten cells that each integrate outputs of local motion-sensitive elements. The integrated postsynaptic signals are conveyed to the presynaptic terminals as graded potentials, superimposed with spikes of variable amplitude (Hengstenberg 1977). VS cells possess large and unique, but overlapping, receptive fields (Hengstenberg et al. 1982; Krapp et al. 1998) and it was previously concluded that they are weakly coupled serially by electrical synapses (Haag and Borst 2004). V1 gets strong input from three VS cells with frontolateral receptive fields and additional weak and potentially indirect input from further VS cells with more lateral receptive fields (Kalb et al. 2006). Although specifications that are typical for chemical synapses are visible at output arborizations of VS cells (Hausen et al. 1980), it is still not known whether individual synapses of the different VS cells to V1 are chemical or electrical. A study by Kalb et al. (2006) revealed that the impact of laser ablation of individual VS cells on V1 spiking activity complied best with electrical synapses between V1 and VS1, but with chemical synapses between the other VS cells and V1.

The costs of publication of this article were defrayed in part by the payment of page charges. The article must therefore be hereby marked "advertisement" in accordance with 18 U.S.C. Section 1734 solely to indicate this fact. 
Synaptic transmission between VS and V1 was recently shown to be almost linear for presynaptic voltage fluctuations up to about 10-20 Hz (Warzecha et al. 2003). This corresponds with the frequency range of motion signals, which is limited because of the low-pass filters inherent to visual motion detection (Warzecha et al. 1998). In addition, presynaptic spikes have a high probability of evoking postsynaptic spikes (Warzecha et al. 2003).

Although visual stimulation as used in previous studies permits a characterization of the functionally relevant operating range of the synapse, it does not allow any assignment of the postsynaptic signal to one specific presynaptic cell because the cells' receptive fields overlap. Moreover, because neuronal responses to identical visual stimuli are highly variable (Warzecha and Egelhaaf 2001), visual stimulation does not provide reproducible presynaptic signals. In addition, visually induced presynaptic voltage changes are limited in their amplitude and their dynamics. We overcame these limitations by controlling the potential of individual VS cells through voltage clamping. This technique guaranteed trial-to-trial reproducibility of the presynaptic potential. It also makes it possible to apply presynaptic voltages exceeding the naturally occurring range in amplitude and dynamics.

\section{METHODS}

\section{Animal preparation and electrophysiology}

Young (1- to 3-day-old) female blowflies (Calliphora vicina) from our laboratory stock were used for the experimental analysis. The animals were waxed to a glass support. Their legs were removed and the wings and abdomen were immobilized with wax. The head was pitched downward and fixed ventrally to the thorax. Subsequently a hole was cut into the back of the head capsule so that both brain hemispheres were accessible and the right and left lobula plate could be viewed from above. Neck muscles were severed. The antennae, some fat bodies, and parts of trachea were removed and the proboscis was stretched out and waxed to the thorax. All wounds were sealed with wax.

V1 was recorded in the left brain hemisphere extracellularly in its output region with borosilicate glass electrodes (GC150TF-10, Clarc Electromedical, Edenbridge, UK) pulled on a DMZ universal puller (Zeitz-Instrumente, Augsburg, Germany). Electrodes were filled with $2 \mathrm{M}$ potassium acetate resulting in a resistance of about $1-5 \mathrm{M} \Omega$. V1 was identified by its sensitivity to downward motion in the frontal part of the visual field contralateral to the recording site in the left half of the brain (Karmeier at al., 2003; Krapp et al., 2001). The signal was amplified using an npi SEC-10 amplifier with an SEC-EXT headstage (npi electronics, Tamm, Germany) in bridged mode. Once a stable V1 recording was established, a presynaptic VS cell (right brain hemisphere) was penetrated in the axon close to its output region using sharp borosilicate glass electrodes (GC100TF-10, Clarc Electromedical) pulled on a Flaming/Brown puller (P97, Sutter Instruments, San Rafael, CA). The electrodes were filled with $2 \mathrm{M}$ potassium acetate and had resistances between 15 and $30 \mathrm{M} \Omega$. The membrane potential was amplified using an npi SEC-10 amplifier with the standard low-voltage headstage (npi electronics).

VS cells were identified in bridged recording mode by their graded depolarizations during presentation of downward motion in their specific receptive field (Krapp et al. 1998). Because of the very similar receptive fields of VS2 and VS3, we did not distinguish between them; the same holds for VS4 and VS5.

The bridged recording mode was then switched to discontinuous single-electrode voltage clamp (dSEVC, duty cycle $1 / 4$, switching frequency about $40 \mathrm{kHz}$ ), and rectangular voltage signals were applied
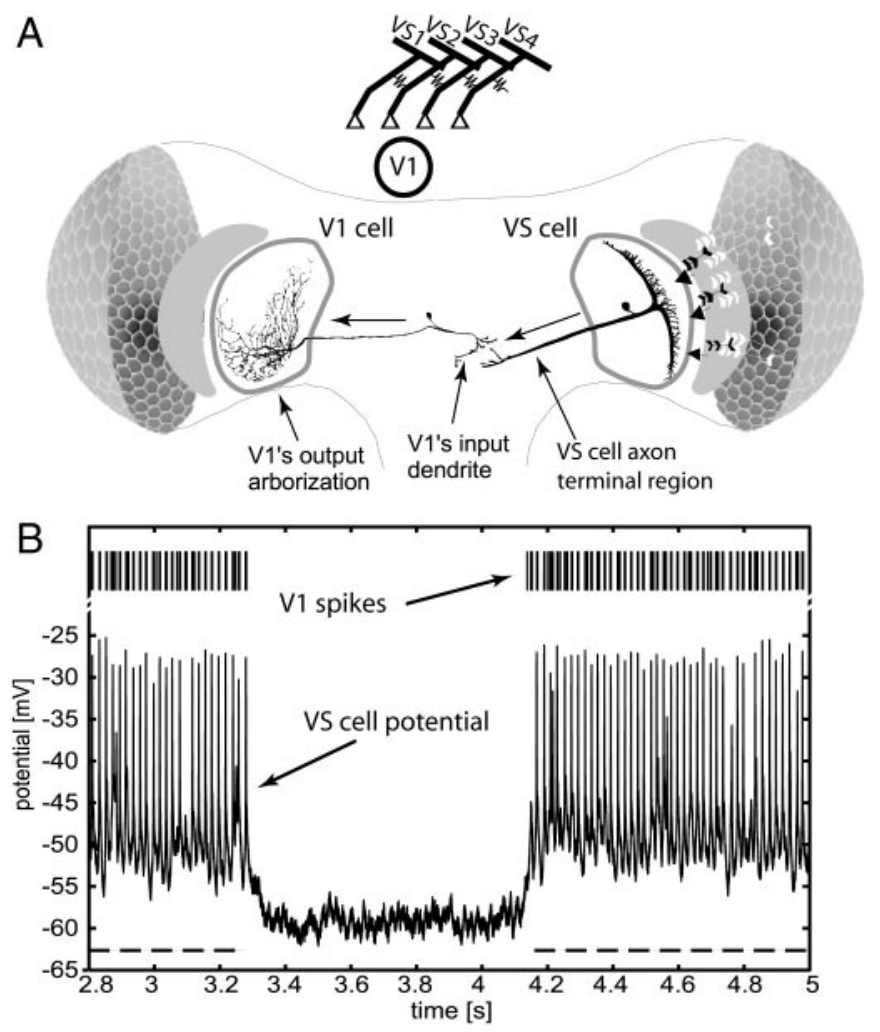

FIG. 1. Synaptic coupling between vertical system (VS) cells and the V1 cell. A: schematic of the fly motion pathway from a caudal perspective (bottom) and the wiring scheme of the VS-V1 circuit (top). Visual system is organized retinotopically in columnar elements. Output of these small-field motion-sensitive elements is pooled by the large dendrites of the VS cells (in this case, the dendrite of a VS2 cell). Signal is conducted to the axon terminal of VS, where it is transferred synaptically to V1 that branches in the contralateral brain hemisphere. Note that the existence of electrical synapses has been proposed at some of the connections instead of purely chemical transmission between VS and V1 (Kalb et al. 2006). VS cells were recorded at the axonal output region. Voltage clamping the cells at this region ensured that the presynaptic voltage of VS was as similar as possible to the signal measured at the voltage-clamp electrode. $B$ : sample dual recording of the presynaptic potential of a VS2/3 cell (bottom trace) and postsynaptic spikes (vertical lines in the top trace) of $\mathrm{V} 1$ during visual stimulation in preferred (indicated by the dotted line) and antipreferred direction (no marker).

to test whether the recording quality was sufficient to provide the required voltage-clamp quality, as checked by careful inspection of the waveform of the amplifier's discontinuous raw output signal. The voltage-clamp recording quality was limited by electrode properties and resulting maximum injectable currents. Thus for each recording, a trade-off had to be found individually between a stable recording (low maximum current injection) and fast-voltage control (high maximum current injection). The recording site close to the synaptic terminals was chosen to minimize space-clamp problems with respect to synaptic output. Note that it was neither intended nor necessary to keep the large, widely ramified dendrites of the neurons under precise voltage clamp. In experiments with visual stimulation, spikes were elicited in VS neurons in addition to the graded depolarizations. Such rapid voltage transients could not be eliminated completely by the voltage clamp and were tolerated as long as their amplitude did not exceed 1-2 $\mathrm{mV}$ (see Figs. $1 B$ and $4, A$ and $B$ ). Note that in the majority of our experiments, no visual stimulation was used and initiation of spikes was not a problem. Recordings with insufficient voltage-clamp quality (instable recording or too much residual membrane potential fluctuation) or too strong cross talk with the extracellular recording were excluded. Dual-recording duration lasted typically 10-30 min. In each experiment, synaptic coupling between V1 
A

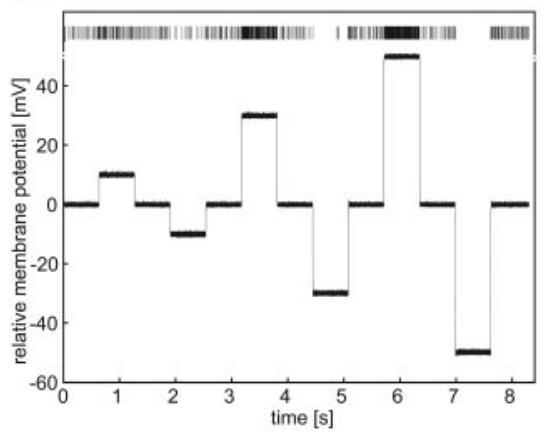

$\mathrm{B}$

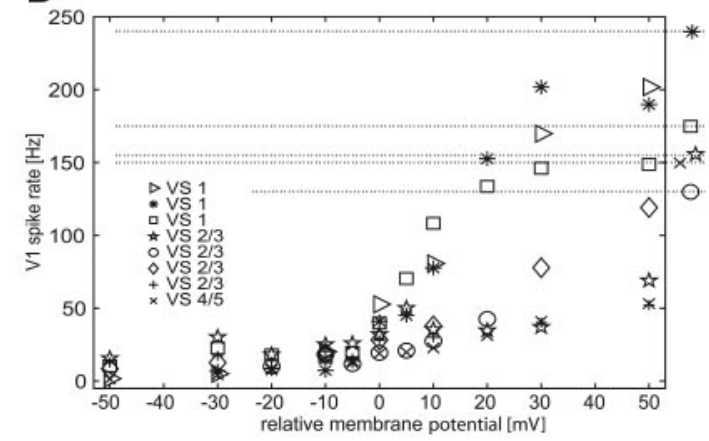

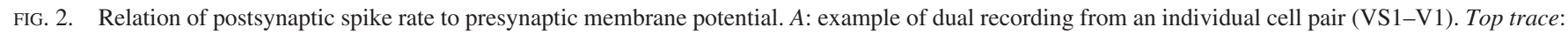

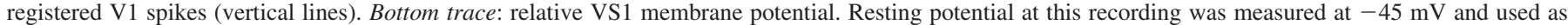

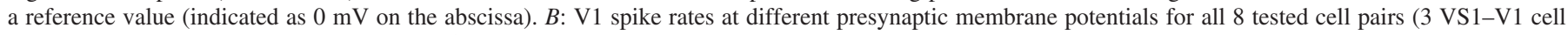

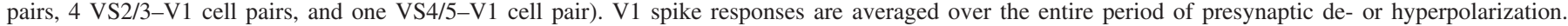

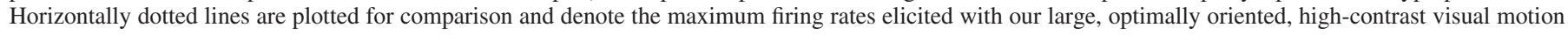

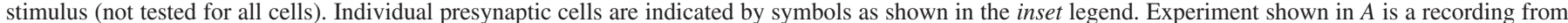
VS1-V1 and marked by the triangle symbol in $B$.

and the VS cell under examination was tested by injecting small currents (about 0.1-0.4 nA) into the VS cell and simultaneously monitoring the $\mathrm{V} 1$ response.

\section{Stimulation and data acquisition}

All data acquisition and stimulation was performed with selfwritten software (Visual C ++6.0 , Microsoft, Redmond, WA) using a standard Intel Pentium4-based personal computer running Windows (Windows 2000 SP4, Microsoft) and a high-precision multifunction I/O card (PD2-MFS-4-500/14, United Electronic Industries, Canton, MA). The extracellular V1 signal, the intracellular VS membrane potential, and the voltage stimulus were each recorded with a sampling frequency of $100 \mathrm{kHz}$. Intracellular recordings were digitized with an amplitude resolution of $0.125 \mathrm{mV}$. Spike detection of the extracellular signal was performed off-line by setting an appropriate threshold.

Visual stimuli consisted of square-wave gratings moving vertically (spatial wavelength: about $17^{\circ}$, temporal frequency: about 1.65 cycles/s, mean luminance: about $500 \mathrm{~cd} / \mathrm{m}$ ). They were provided by a custom-made LED matrix in front of the fly's head covering a visual field from about -20 to about $+45^{\circ}$ horizontally and from about +20 to $-30^{\circ}$ vertically.

The voltage-clamp experiments used two different sets of voltage commands. The first consisted of a set of rectangular potential steps each lasting $600 \mathrm{~ms}$ (in some experiments, all durations were prolonged to $1,200 \mathrm{~ms}$ ). These steps were provided in two application protocols, one applying a sequence of voltage steps to $+5,-5,+10$, $-10,+20$, and $-20 \mathrm{mV}$ relative to the resting potential. Each step was followed by a 600-ms (or 1,200-ms) duration of clamping the voltage at the resting potential. The second application protocol had an identical time course, but the potential steps were different: +10 , $-10,+30,-30,+50$, and $-50 \mathrm{mV}$. The rise time of the voltage steps was allowed to take $1 \mathrm{~ms}$ to reach $80 \%$ of the clamp potential and 3 ms to reach a stable steady state.

For the second set of voltage commands, the voltage was driven with sinusoidal time courses using frequencies of 1, 2, 4, 8, 16, 32, 42, and $64 \mathrm{~Hz}$ in pseudorandom order and peak amplitudes of \pm 12 or $\pm 20 \mathrm{mV}$. Each frequency was clamped for $1 \mathrm{~s}$ followed by $300 \mathrm{~ms}$ when the potential was clamped to the resting potential.

All data analysis was performed off-line using custom analysis routines written in MatLab (The MathWorks, Natick, MA).

\section{R E S U L T S}

Synaptic signal transfer was studied in a visual motionprocessing region of the fly brain using simultaneous dual recordings from a presynaptic VS neuron and the postsynaptic V1 neuron (recording site sketched schematically in Fig. 1A; dual recording example shown in Fig. $1 B$ ). The VS cells are predominantly sensitive to vertical motion (Hengstenberg et al. 1982). During downward (preferred direction) and upward (null direction) visual motion in their receptive fields, VS cells showed strong graded de- and hyperpolarizing axonal membrane potential responses. With amplitudes of $\leq 10-15 \mathrm{mV}$ relative to the resting potential, the depolarizations were somewhat stronger than the hyperpolarizations during motion in the null direction that never exceeded potential shifts of $-10 \mathrm{mV}$. Spikes with variable amplitude reaching about $40 \mathrm{mV}$ relative to the resting potential were superimposed on the graded depolarization at rates of up to about $60 \mathrm{~Hz}$ (see bottom trace in Fig. $1 B$ ) during motion in the preferred direction. The V1 cell is postsynaptic to several VS cells and responds to downward motion with spikes correlated to the VS activity (Warzecha et al. 2003) (example shown in Fig. 1B). The following experiments were performed to systematically investigate the synaptic transfer properties underlying this correlation on the basis of single VS-V1 synapses.

\section{Signaling range, gain, and linearity of individual VS/V1 synapses}

In the first set of experiments, we characterized the transmission properties of individual synaptic connections by monitoring postsynaptic responses to precisely controlled membrane potential deflections of a single presynaptic cell. During the absence of any visual stimulation, one VS cell was clamped to various membrane potentials (example shown in Fig. 2A). With clamped potential changes of maximum $\pm 50 \mathrm{mV}$ relative to the resting potential [which had a mean of $-43 \mathrm{mV}$ with a $\mathrm{SD}$ of $\pm 8 \mathrm{mV}(n=9)]$, these values exceeded by far the range of graded potential fluctuations that can be evoked by visual motion stimulation. The latter cover a range of only about 15 $\mathrm{mV}$ for depolarizations and about $-10 \mathrm{mV}$ for hyperpolarizations. Depolarizations reached about $50 \mathrm{mV}$ above resting potential only when spikes occurred. These were very transient, lasting $<1 \mathrm{~ms}$. Thus presynaptic voltage clamping made it possible to test synaptic transmission both within and beyond the natural operating range. 
Figure $2 A$ shows a sample V1 spike train during voltage clamping of the membrane potential of a VS1 neuron with deand hyperpolarizing values of increasing amplitude (de- and hyperpolarization of $\leq 50 \mathrm{mV}$ relative to the resting potential). V1 spike activity was already raised above baseline level at the weakest presynaptic depolarization of $+10 \mathrm{mV}$ relative to the resting potential and remained above baseline activity over the entire 600-ms period of the tonic depolarizing voltage command. Presynaptic depolarizations of increasingly higher amplitude induced increasingly stronger postsynaptic spike activity. Similar to presynaptic depolarizations, hyperpolarizations of -20 and $-50 \mathrm{mV}$ differed in their impact on postsynaptic activity, even though naturally occurring hyperpolarization in VS neurons never exceeded $-10 \mathrm{mV}$. Figure $2 B$ shows postsynaptic firing rates in response to various presynaptic clamp potentials for eight dual recordings (three VS1, four VS2/3 cells, and one VS4/5 cell). Additionally, the postsynaptic response level obtained with visual stimulation was determined for four of the V1 neurons and is plotted in Fig. $2 B$.

The impact on V1 spiking activity of the presynaptic voltage clamp of different VS neurons to various holding levels differed considerably between recordings. In some recordings, strong presynaptic depolarization was necessary to raise V1 activity above baseline levels (e.g., five-point star or cross symbols in Fig. 2B), whereas in other recordings, even with moderate presynaptic depolarization, V1 responded with high activity (e.g., square symbols in Fig. 2B). Regardless of these differences in coupling strength, the responses to presynaptic depolarizations remained linear over a wide range of presynaptic depolarizations. In all experiments, the relationship between presynaptic depolarization and $\mathrm{V} 1$ response was linear for depolarizations of up to $\geq 20 \mathrm{mV}$. In some experiments, the input-output function remained linear up to the maximum depolarization applied.

It is evident that these differences were correlated with a different overall gain in the individual input-output functions. In those recordings in which the gain was rather low, the linearity was retained over the entire probed range of depolarizations. A nonlinearity of the input-output characteristic was introduced only when, because of its high gain, the V1 response reached spike rates similarly high to those obtained using strong visual stimuli (compare thin dotted lines in Fig. $2 B$ ). Both results-input-output linearity exceeding the range of naturally occurring depolarizations and maintenance of linearity up to near-maximum visually inducible spike ratessuggest that linearity of transmission is not limited by constraints of VS-V1 synapses themselves. Instead, V1 has an intrinsic maximum spike generation rate that limits the further linear slope of the VS-V1 transfer function.

Although a clear identification of cell types was often problematic, and definitive conclusions would require larger numbers of recordings, the synaptic gain seems to depend on the particular type of presynaptic VS cell. Depolarization of the VS1 cells (Fig. 2B, triangle, square, and six-pointed star symbols) led to the largest firing rates in $\mathrm{V} 1$, whereas the gain of the VS2/3-V1 connection was lower. The gain in the VS4/5-V1 connection was found to be even weaker. Accordingly, depolarization of a VS4/5 cell (" $\times$ " symbol in Fig. 2B) resulted in a small, but still detectable, response in the V1 spike rate. This apparently weak influence may suggest an indirect coupling of VS4/5 by VS2/3 (to which VS4/5 was found to be

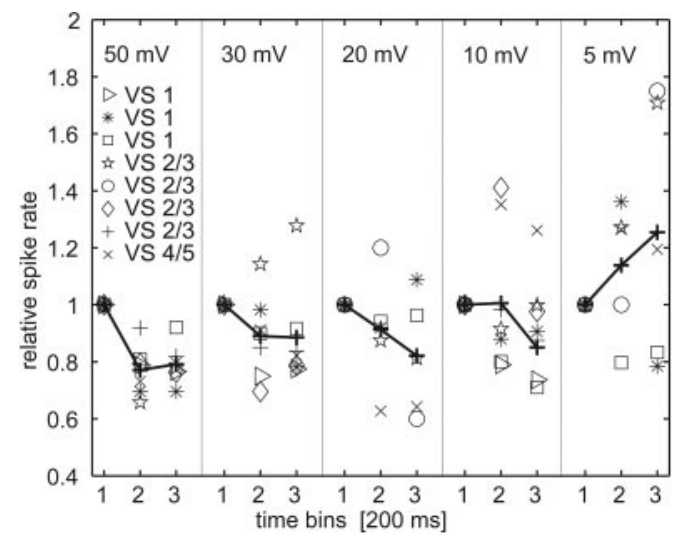

FIG. 3. Course of V1 spike rates during continuous presynaptic depolarization. Same data as in Fig. $2 B$ are used here. Each 600-ms depolarization was divided into 3 equal time bins and the spikes occurring were counted for each bin and normalized to the first bin. Thick lines represent mean values calculated from all cells; data from individual cells are indicated by different symbols (same assignment as in Fig. 2).

coupled electrically; see Haag and Borst 2004, 2005). However, a weak direct synaptic coupling between VS4/5 and V1 is also compatible with the experimental results. Again, hyperpolarization of VS1 cells had a stronger postsynaptic effect than VS2/3, whereas VS4/5 had only a weak impact on V1.

In conclusion, two aspects are remarkable with respect to synaptic signaling. First, for all VS cells, it was a common observation that presynaptic depolarization within the natural range of graded membrane potential changes $(\leq 10-20 \mathrm{mV})$ resulted in an almost linear increase in the $\mathrm{V} 1$ spike response. The linearity of VS-V1 signal transfer was maintained until V1 reached spike rates comparable to those elicited by strong visual stimuli, even when the presynaptic cell considerably exceeded the naturally occurring range of depolarization. Second, depolarization of only a single presynaptic cell was able to drive V1 to high, sustained spike rates reaching the same frequency as obtained by visual stimulation. Note that during visual stimulation, multiple VS cells were excited simultaneously and thus V1 always received excitatory input from at least three cells.

\section{Transient properties of V1 activity during sustained presynaptic depolarization}

Although, at first sight, there was no obvious decrease in postsynaptic spike rate during constant presynaptic depolarization (Fig. 2A), we still analyzed this issue systematically. We scrutinized the time courses of V1 spike rates during sustained presynaptic depolarization. Figure 3 shows V1 spike rates in subsequent 200-ms time bins during presynaptic voltage clamp to different depolarization levels for all cells tested. Spike activity was normalized to the mean activity in the first $200 \mathrm{~ms}$ of each trial. Mean values were calculated for individual cells and these were pooled to obtain mean values over all cells (thick lines in Fig. 3). With the exception of the spike rate at $5-\mathrm{mV}$ depolarization, the mean values generally showed a slight decrease over time.

Because the variability of the spike rate over time was high, the significance of the spike rate decrease was controlled with a Wilcoxon signed-ranks test (null hypothesis: the spike rate of the first $200 \mathrm{~ms}$ will not be significantly higher than the spike 
TABLE 1. V1 spike rates during continuous presynaptic depolarization averaged over consecutive time intervals

\begin{tabular}{cccc}
\hline \hline & & \multicolumn{2}{c}{$P$ Value for the } \\
\cline { 3 - 4 } $\begin{array}{c}\text { Depolarization, } \\
\mathrm{mV}\end{array}$ & $\begin{array}{c}\text { Number of } \\
\text { Trials }\end{array}$ & Second Time Interval & Third Time Interval \\
\hline 5 & 5 & 0.2500 & 0.4375 \\
10 & 8 & 0.5469 & 0.0781 \\
20 & 5 & 0.4375 & 0.1875 \\
30 & 7 & 0.1094 & 0.2969 \\
50 & 7 & 0.0156 & 0.0313 \\
\hline
\end{tabular}

Results of Wilcoxon signed-ranks tests. $P$ values measure evidence against the null hypothesis (the spike rate of the first $200 \mathrm{~ms}$ will not be significantly higher than the spike rates of the subsequent second and third time intervals).

rates of the subsequent time slots). For a 5-mV depolarization $(n=5)$, we obtained a probability value of $P=0.25$ for the second $200 \mathrm{~ms}$ and $P=0.438$ for the third $200 \mathrm{~ms}$, whereas during the $50-\mathrm{mV}(n=7)$, depolarization, values were $P=$ 0.016 for the second and $P=0.031$ for the third $200-\mathrm{ms}$ bin. All statistical findings are reported in Table 1. Thus there was a slight tendency for V1 to decrease its response during sustained depolarizing synaptic input. This spike rate attenuation might result either from synaptic mechanisms or from mechanisms intrinsic to V1 such as fatigue during spike generation.

\section{Impact of functionally inactivating one presynaptic VS cell on V1 visual motion response}

In the following experiments, we examined how V1 integrates the output from its presynaptic VS cells. We asked whether the lack of input from one presynaptic cell could be compensated by input from the others or whether simultaneous input from all presynaptic elements is required to generate a response in V1, as would be the case if input integration were highly cooperative. We compared the mean V1 spike rate during visual stimulation under normal conditions with a condition in which one VS cell was clamped at its output region to the resting potential (Fig. 4). The sustained graded component of the motion response of the VS neuron could be eliminated completely by the voltage clamp and spikes were reduced to small fluctuations of about $1 \mathrm{mV}$ in amplitude. Without the presynaptic voltage clamp, motion in the preferred direction elicited a V1 response of maximum firing rates up to nearly $250 \mathrm{~Hz}$ and null-direction motion led to complete inhibition of spiking. When one presynaptic cell was voltage clamped to its resting potential and thus functionally deleted from the circuit, the V1 spike rate during preferred-direction motion decreased by $\leq 40 \%$, and occasional spikes were elicited during antipreferred-direction motion (Fig. 4B). Although the reduction in spike activity was usually smaller than that in this example (Fig. $4 C$ ), it was present in all experiments when VS1 or VS2/3 had been clamped. These experiments showed that it was not necessary for V1 to receive input from all VS cells simultaneously. Instead, visual motion input was able to drive V1 to a high spike rate even when the input from one cell was absent or at least reduced to very small transient depolarizations (1-2 $\mathrm{mV})$.

Different results were obtained when VS4/5 was clamped. Although a coupling of VS4/5 to V1 could be demonstrated by current injection, the V1 motion response remained unchanged regardless of whether VS4/5 had been clamped to its resting potential (data not shown). This finding corroborates previous findings (Kalb et al. 2006), indicating that more lateral VS cells, such as VS4, have less influence on V1 activity than more frontal VS cells.

\section{Dynamic properties of synaptic transmission}

Dynamic properties of synapses are functionally relevant because they may endow synapses with the ability to filter their input signals in a specific way. Along with other functions, the separation of signal from noise and novelty detection has been concluded to be based on specific synaptic temporal properties (e.g., Field and Rieke 2002; Juusola et al. 1995; Luksch et al. 2004; Rose and Fortune 1999).

The decrease of V1 activity during constant presynaptic depolarization described earlier (see Fig. 3) might indicate weak high-pass filter properties of the VS-V1 synapses. To investigate these properties more systematically, we presynaptically applied sine-wave potentials covering a frequency range from 1 to $64 \mathrm{~Hz}$ (see Fig. 5A for a sample recording). First, we examined the mean spike count of the V1 response as a function of the frequency of presynaptic membrane potential modulations. Figure $5 B$ shows the mean spike rates during application of the different frequencies for all probed cells. To exclude an influence of reductions of the spike rate during
A

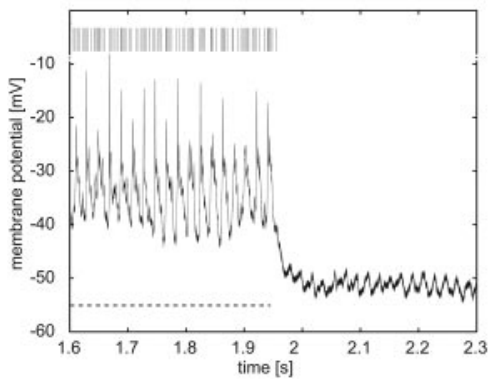

B

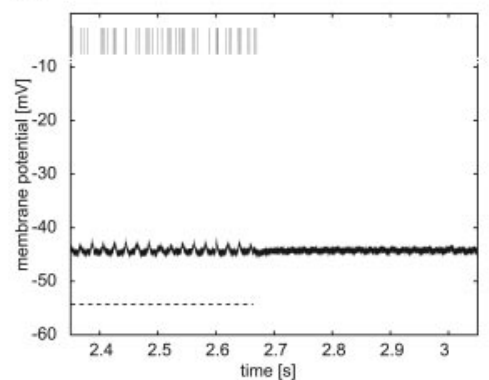

C

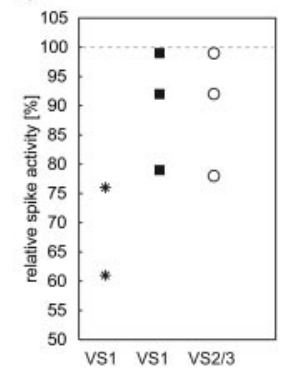

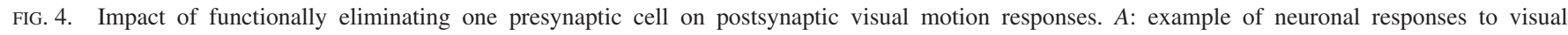

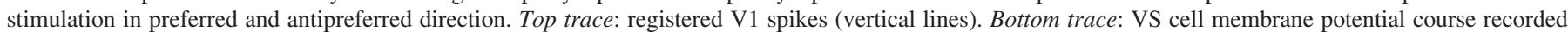

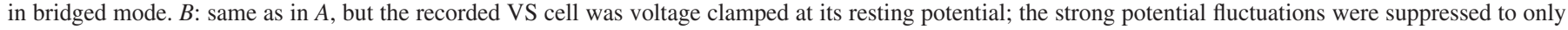

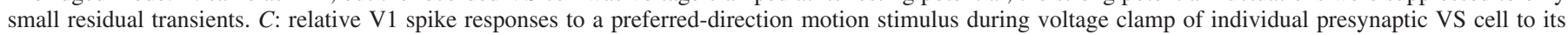

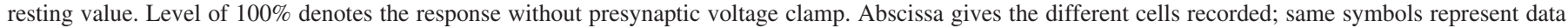

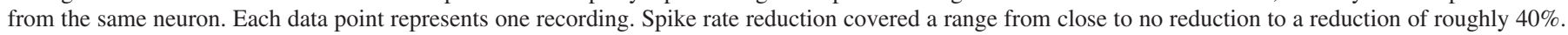


A

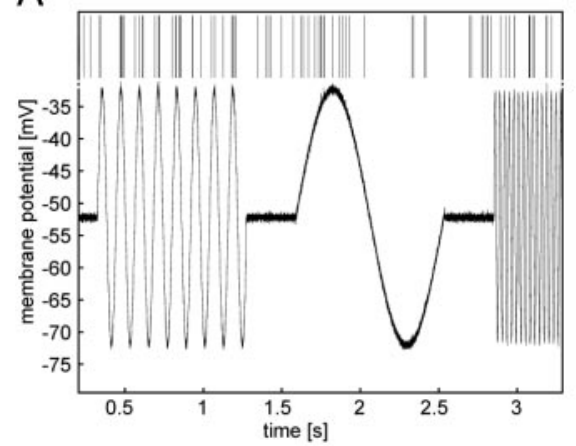

B

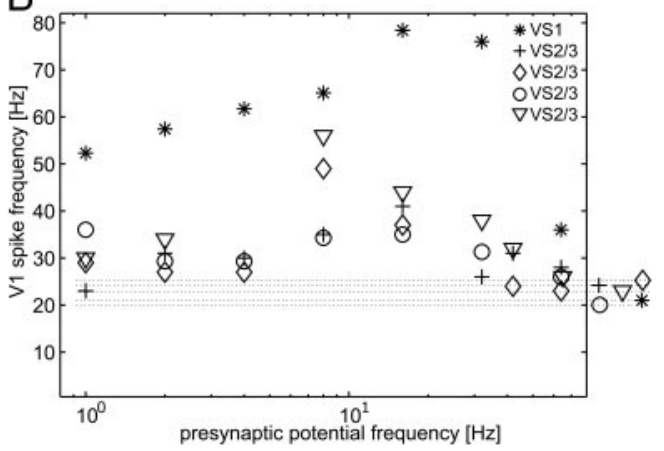

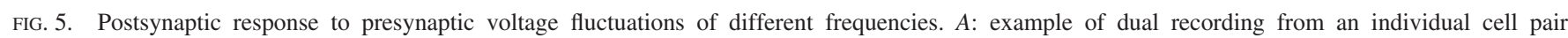

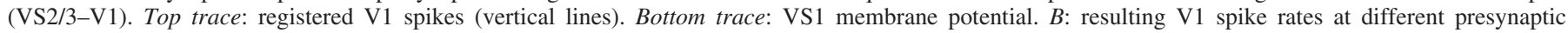

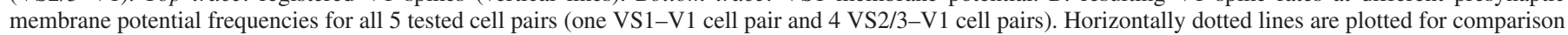

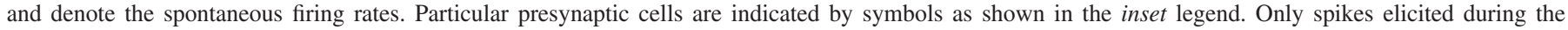

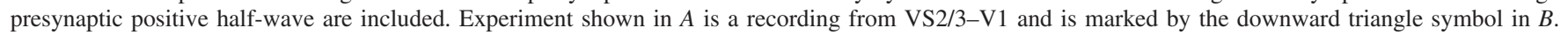

presynaptic hyperpolarization, we evaluated only the depolarizing half waves $(0-\pi)$. For comparison, the spontaneous spike rates are also plotted in the figure (thin horizontal lines). Initially, the spike count increased slightly with increasing frequency of presynaptic voltage modulations. For all analyzed cells, it peaked consistently at 8 or $16 \mathrm{~Hz}$ and fell off at higher frequencies. Thus synaptic transfer revealed a weak band-pass characteristic. Similar results were obtained when a period of $0-1.4 \pi$ was evaluated to account for delays in spike generation.

The range of V1 response amplitudes was large and seemed to depend on the type of voltage-clamped presynaptic VS cell, rather like the dependencies found in the experiments using rectangular-clamp protocols (see above). Whereas V1 reached firing rates of $\leq 80 \mathrm{~Hz}$ when stimulating VS1, the spike rate remained in the range of $30-50 \mathrm{~Hz}$ when a VS2/3 cell had been stimulated (no VS4/5 cell was tested with the dynamic-clamp protocol because the postsynaptic responses obtained in the experiments described above were only very weak).

As can be seen in the recording trace in Fig. $5 A$, there was some coupling of V1 spikes to the phase of the presynaptic VS cell membrane potential. To investigate whether the temporal coupling of V1 spikes to the phase of the presynaptic sinewave potential changed with presynaptic frequency, we plotted the spike rate as a function of the phase irrespective of the frequency of the sinusoidal potential modulations. The spike response to each frequency was normalized to the response in the $8-\mathrm{Hz}$ condition for each trial. Then, the sine waves were binned into $0.1 \pi$ intervals and all spikes of the same phase were summed separately for each tested frequency. Figure 6 shows such plots for all data separately for VS1 (single cell, subplots $A, C, E$ ) and VS2/3 (average over four cells, right column of subplots).

A phase coupling of postsynaptic spikes to the presynaptic membrane potential modulation was clearly present in the VS1-V1 recording, but less pronounced in VS2/3-V1 recordings. The phase dependency of the V1 spike response to VS1 (left column of Figure, subplots $A, C, E$ ) reflected the time course of the presynaptic clamped sine potential reasonably well. For all frequencies, the maximum spike rate was at the sine-wave vertex in the range of about $0.4-0.7 \pi$. At the higher frequencies of 32 and $64 \mathrm{~Hz}$, the spike response maxima became narrower and appeared to be coupled more precisely to the presynaptic membrane potential vertex. The phase of the response was shifted toward $0.6-0.7 \pi$.

The spike response of V1 to stimulation of VS2/3 (right column of Fig. 6) was similar, but not coupled as precisely to the presynaptic sine phase as during VS1 stimulation. This may be attributable to a lower overall synaptic gain (compare Fig. $5 B)$. With increasing frequency, the coupling became more distinct and there was also some phase shift at the high frequencies. Whereas at frequencies $<32 \mathrm{~Hz}$, the maximum was located at $0.4-0.6 \pi$ of the presynaptic voltage sine wave, it appeared to shift to $0.8-1.1 \pi$ at frequencies $\geq 32 \mathrm{~Hz}$.

\section{I S C U S S I O N}

Synaptic signal transfer has been studied in pairs of motionsensitive neurons already identified in the fly. We examined the synaptic signal transmission properties systematically by using simultaneous dual recordings and the voltage-clamp technique to control the potential in the presynaptic cell's output region. Unlike previous studies in which visual stimulation was used to characterize these synapses (Kurtz et al. 2001; Warzecha et al. 2003), our approach allows us to investigate individual synaptic connections during transmission of signals, which are virtually identical from trial to trial. Moreover, it is possible to examine synaptic transmission beyond the operating range of the system under natural conditions, that is, during visualmotion stimulation.

\section{Synaptic connections contribute individually to the motion response}

Our experiments show that VS cells contribute individually to the motion response of V1 and that the postsynaptic cell can be driven by a single input element to sustained spike activity at high frequencies.

Functionally eliminating one presynaptic cell during visualmotion stimulation reduces, but does not abolish, the response of V1. This makes it seem unlikely that presynaptic elements need to be simultaneously active to induce postsynaptic activity. If this were the case, inactivating individual presynaptic inputs would more severely affect the capacity of the postsynaptic cell to respond to visual motion. Instead, our observations suggest a sublinear integration of individual synaptic inputs by 
A

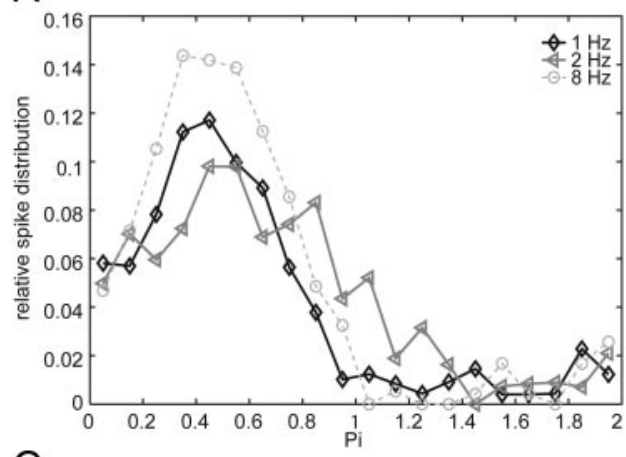

C

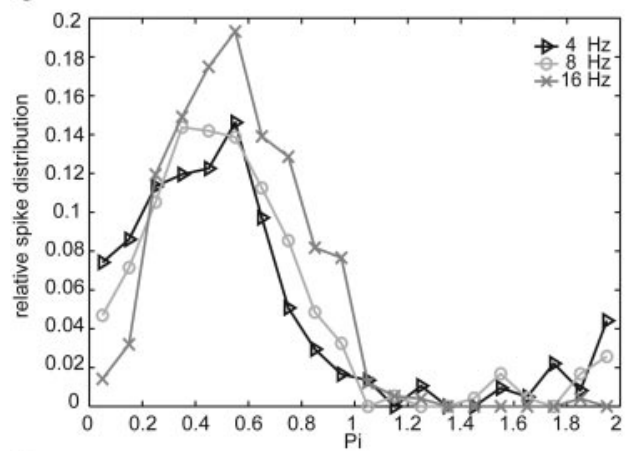

E

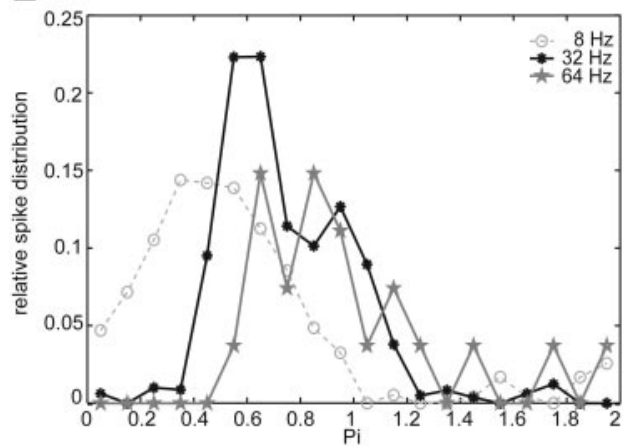

B

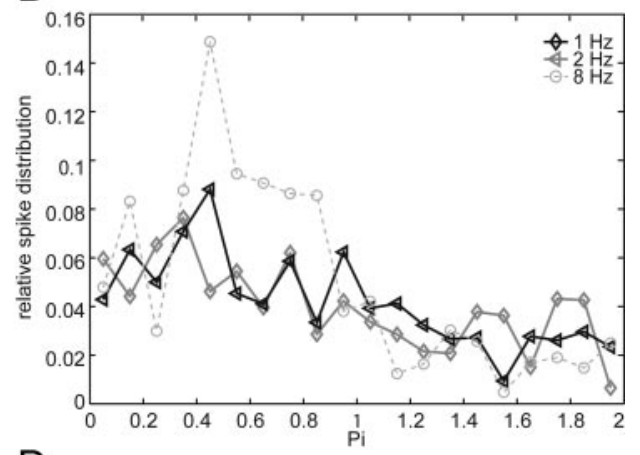

$\mathrm{D}$

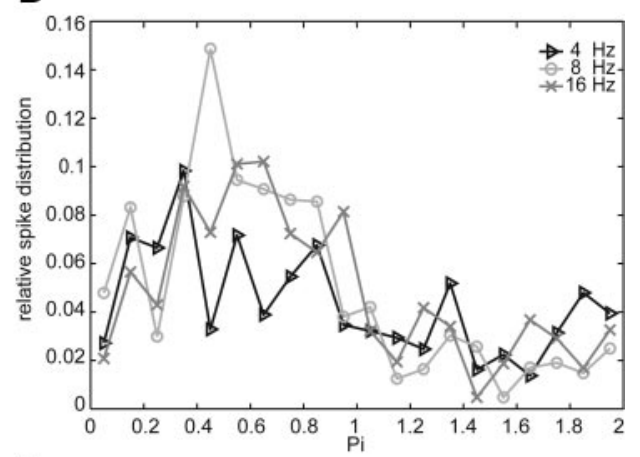

$\mathrm{F}$

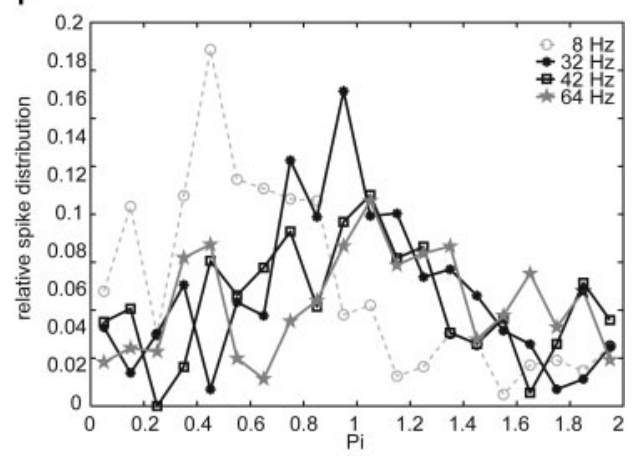

FIG. 6. Relation between postsynaptic spikes and the phase of the presynaptic potential. Left column (subplots $A, C, E$ ) shows results for VS1-V1 data (one cell with 7 trials for the 1 - to $16-\mathrm{Hz}$ condition, 6 trials for the $32-\mathrm{Hz}$ condition, and one trial for the 64-Hz condition). Right column (subplots $B$, $D, F$ ) for $\mathrm{VS} 2 / 3-\mathrm{V} 1$ data (4 cells with 12 trials for the $1-$ to $32-\mathrm{Hz}$ condition, 10 trials for the $32-\mathrm{Hz}$ condition, 6 trials for the $42-\mathrm{Hz}$ condition, and 12 trials for the $64-\mathrm{Hz}$ condition). All results were normalized to the $8-\mathrm{Hz}$ condition (open circle symbol, shown in all subplots). the V1 neuron because the decrease of postsynaptic motion responses after elimination of one presynaptic element does not exceed one third of the activity of the unaffected pathway. This is in agreement with a recent laser-ablation study on the same synaptic circuit from Kalb et al. (2006). They found that coding of white-noise velocity fluctuations by $\mathrm{V} 1$ did not deteriorate when single VS2/3 neurons were killed. It must, however, be considered that application of the voltage-clamp technique does not allow us to completely suppress spikes elicited during visual stimulation. Thus a residual influence of the clamped cells on V1 activity cannot be excluded entirely.

\section{Mixed potential synapses conveying spike-mediated and graded presynaptic signals}

Unlike most synapses studied so far (but see Alle and Geiger 2006; Graubard et al. 1980; Ivanov and Calabrese 2003; Shu et al. 2006; Simmons 1999), the presynaptic signal at VS-V1 synapses consists of two components: a rather slow process of graded potential changes and a fast component of spikes superimposed on the graded potential. This mixed potential mode confronts the synapses with the problem of having to deal with two types of signal requiring different physiological adaptations to function optimally. In terms of amplitudes, graded signaling may manage with a much smaller synaptic operating range than spikes, and achieve the highest possible resolution when the synaptic operating range matches exactly that of the graded presynaptic voltage fluctuations. Moreover, temporal low-pass filtering may increase the signal-to-noise ratio of the transmission of graded signals because information on fluctuations in visual-motion velocity is limited to frequencies $\$ 20 \mathrm{~Hz}$ (Warzecha et al. 1998). Such low-pass filtering would, however, hamper spike-mediated transmission. In general, this problem could be solved by segregating fast and slow synaptic transmission to spatially dislocated synaptic release sites like those Meinrenken et al. (2002) proposed might be present at the calyx of Held in rat auditory brain stem. At shared release sites, the coexistence of different $\mathrm{Ca}^{2+}$ channels-fast high-voltage-activated on the one hand and slow low-voltage-activated on the other hand-might help to transfer both fast and slow membrane components (Ivanov and Calabrese 2006) into transmitter release. For nerve cells in the rat's auditory system, Awatramani et al. (2005) showed that 
changes of the presynaptic background $\mathrm{Ca}^{2+}$ level induced by slow shifts of the membrane potential modify the magnitude of fast action potential-mediated transmitter release. Thus both tonic and phasic membrane potential changes are expressed in the regulation of transmitter release.

\section{The working range of VS-V1 synapses for sustained signal transmission is broad}

By directly driving only single presynaptic cells through the use of voltage clamping, we have managed to show that purely graded presynaptic voltage signals without any superimposed spikes are sufficient to induce high postsynaptic spiking activity. Furthermore, we have corroborated the notion of linear or slightly sublinear integration of the different presynaptic cells by the postsynaptic neuron. However, the inputs from different VS cells are weighted differently by V1: depolarizations of VS1 lead to a higher V1 spike rate, whereas VS2/3 or VS4/5 depolarizations lead to lower spike rates. This is again in agreement with the impact of laser ablation of presynaptic VS neurons on V1, which have been reported to be strongest for VS1 and weakest for VS4/5 (Kalb et al. 2006).

We have shown that the signal transmission at single VS-V1 synapses follows a linear function over a wide range of presynaptic depolarizations and becomes nonlinear only when the postsynaptic spike rate reaches maximum values. It is surprising to see that linearity is maintained over a much wider presynaptic range than that likely to occur for graded responses to visual-motion stimulation. This finding seems to contradict the expectation that the synaptic signaling range will be exploited optimally when the limited range of high input-output gain is matched to the naturally occurring input activities. However, it has to be considered that, in addition to sustained graded voltage signals, transient spikes (which superimpose the graded depolarizations) are also transmitted synaptically (Warzecha et al. 2003). A large proportion of presynaptic depolarization transients elicit postsynaptic spikes within a 2-ms time window. These presynaptic spikes often reach amplitudes similar to the most positive clamp potentials in our experiments, although, in most cases, for only $<1 \mathrm{~ms}$. Transmission of spikes would not require the synapse to maintain high-level activity over an extended period of time. Thus the mixed potential nature of the synapse can only in part provide a reason for the large amplitude range of synaptic signaling. Moreover, it is not known whether spikes in V1 are preferentially triggered by a simultaneous occurrence of spikes in several presynaptic VS cells.

\section{Postsynaptic activity is maintained during sustained presynaptic depolarization}

We have found that during prolonged presynaptic depolarization, regardless of its amplitude, the postsynaptic spike rate remains high for the entire stimulation time with only a slight attenuation. Assuming the synaptic connection between VS and V1 is chemical, and that signal transfer is mediated by transmitter release, this would require mechanisms preventing transmitter pool depletion, as found in specialized graded synapses such as ribbon synapses in vertebrate retina (DeVries and Baylor 1993; Sterling and Matthews 2005). Note that, even here, transmitter depletion is an issue, at least when the natural amplitude range is exceeded (von Gersdorff and Matthews 1999). For the VS-V1 synapse, our results may be explained more easily by assuming electric coupling. Although there is no direct experimental evidence in favor of this hypothesis, it has been concluded that electrical coupling between VS1 and V1 (but chemical synapses between other VS cells and V1) provides the best explanation of results obtained after laser ablation of individual VS cells (Kalb et al. 2006). This correlates with the higher gain for VS1-V1 connections compared with the other synaptic connections (see Fig. $2 B$ ) and might suggest that inputs by electrical synapses have a higher impact on V1 activity than chemical synaptic inputs. Note, however, that in the present study, apart from different synaptic gain, synaptic properties such as the amplitude and dynamic working range do not differ systematically between individual VS cells. Arguments in favor of chemical synapses are the existence of specializations typical for chemical synapses in the output region of VS (Hausen et al. 1980); a close correlation between presynaptic voltage, presynaptic $\mathrm{Ca}^{2+}$ signals, and V1 spiking activity (Kurtz et al. 2001); and the absence of dye-coupling between VS and V1 (Haag and Borst 2005). The latter finding could, however, be explained by the thin diameter of V1 dendritic branches, which may be difficult to discern with conventional microscopic methods. Dual intracellular recordings with current injection into V1 could help to fully resolve the nature of VS-V1 synapses, but, because of their high technical demand, have not yet been performed.

V1 spike rate decreases slightly during sustained presynaptic depolarization. Possible reasons for this decrease may be transmitter depletion (for review see von Gersdorff and Matthews 1999) at VS-V1 synapses or spike-frequency adaptation (Benda and Herz 2003) in V1. Although the decline in V1 spike rate during sustained presynaptic depolarization is weak, it may be relevant in the context of motion adaptation, that is, in processes leading to a gradual attenuation of the responses of fly TCs during long-lasting motion stimulation (Harris et al. 2000; Maddess and Laughlin 1985). Currently, there is evidence that these processes are located both in the input circuitry of TCs and in TCs themselves. In the present study, the response of V1 declines even when the membrane potential of VS is kept on a constant depolarized level. This shows that an additional mechanism leading to motion adaptation is effective either at the output synapse of TCs or downstream in the postsynaptic neuron. Thus motion adaptation appears to be a multilevel phenomenon occurring on all relevant stages along the motion-vision pathway.

Whether this effect is caused by fatigue or arises from a specific adaptation process such as activity-regulated changes in membrane properties cannot be determined on the basis of the present results.

\section{Dynamic signal transmission at the VS-VI synapses follows weak band-pass characteristics}

Imposing sine-wave voltage modulations on one presynaptic VS neuron reveals that VS-V1 synapses have a high gain over a large spectrum of frequencies. As indicated by our results (compare Fig. 5B), dynamic transmission at this synapse has a weak band-pass characteristic.

Previously it was shown that during visual stimulation with white-noise velocity fluctuations, this synapse transmits fre- 
quencies up to about $10-20 \mathrm{~Hz}$ linearly and reliably-that is, the frequency range thought to be most important from a functional perspective (Warzecha et al. 2003). However, directly manipulating the presynaptic potential by voltage clamp suggests that the dynamic range of the synapse considerably exceeds the range of frequencies transmitted by the visual motion pathway. This extended frequency range of synaptic signaling may reflect the ability of the VS-V1 synapse to transmit not only graded membrane potential changes but also spikes. In contrast to the weak band-bass characteristic of the VS-V1 synapse, synaptic transmission in the locust ocellar system showed strong high-pass properties (Simmons 2002). Instead of resulting directly from synaptic properties, the band-pass characteristic measured in the present study may well be intrinsic to the membrane properties of V1 (cf. Borst and Haag 1996; Haag and Borst 1996).

With increased frequency of presynaptic sine-wave depolarizations, the postsynaptic spikes are coupled more precisely to the presynaptic signal. A similar time-locking of spikes to fast sinusoidal membrane potential fluctuations was observed in an integrate-and-fire model of another fly tangential neuron (Kretzberg et al. 2001). The observed increased phase shift of the postsynaptic response with increasing presynaptic stimulation frequency does not provide evidence for a frequencydependent latency. Instead, it may be the result of a constant temporal delay, which would cause larger phase shifts during fast fluctuation in which the period length is short.

In summary, our results are in agreement with a linear or slightly sublinear summation of several differently weighted presynaptic inputs by V1 with a saturation limit set by V1 maximum spike rate. Single VS-V1 synapses cover a larger working range both in terms of steady-state and dynamic presynaptic depolarizations than that expected from naturally occurring graded presynaptic visual motion signals. As a functional consequence, the synapses can handle the special properties of the presynaptic VS cell signals that consist of signals with a distinctly different frequency content-that is, graded potentials that have most of their power during visual stimulation up to only $10-20 \mathrm{~Hz}$ and fast spikelike signals that also contain much higher frequencies. In the future, recording V1 activity during fast, action potential-like waveform commands in VS neurons could help to further resolve the functional significance of presynaptic signals with different time courses.

\section{REFERENCES}

Alle H, Geiger JR. Combined analog and action potential coding in hippocampal mossy fibers. Science 311: 1290-1293, 2006.

Arbas EA, Calabrese I. Slow oscillations of membrane potential in interneurons that control heartbeat in the medicinal leech. J Neurosci 12: 39533960, 1987.

Awatramani GB, Price GD, Trussell LO. Modulation of transmitter release by presynaptic resting potential and background calcium levels. Neuron 48 : 109-121, 2005.

Benda J, Herz AV. A universal model for spike-frequency adaptation. Neural Comput 15: 2523-2564, 2003.

Borst A, Haag J. The intrinsic electrophysiological characteristics of fly lobula plate tangential cells: I. Passive membrane properties. J Comput Neurosci 3: 313-336, 1996.

DeVries SH, Baylor DA. Synaptic circuitry of the retina and olfactory bulb. Cell 72: 139-149, 1999.

Field GD, Rieke F. Nonlinear signal transfer from mouse rods to bipolar cells and implications for visual sensitivity. Neuron 34: 773-785, 2002.

Graubard K, Raper J, Hartline DK. Graded synaptic transmission between spiking neurons. Proc Natl Acad Sci USA 77: 3733-3735, 1980.
Haag J, Borst A. Amplification of high-frequency synaptic inputs by active dendritic memebrane processes. Nature 379: 639-641, 1996.

Haag J, Borst A. Neural mechanism underlying complex receptive field properties of motion-sensitive interneurons. Nat Neurosci 7: 628-634, 2004.

Haag J, Borst A. Dye-coupling visualizes networks of large-field motionsensitive neurons in the fly. J Comp Physiol A Sens Neural Behav Physiol 191: 445-454, 2005.

Haag J, Vermeulen A, Borst A. The intrinsic electrophysiological characteristics of fly lobula plate tangential cells: III. Visual response properties. J Comput Neurosci 7: 213-234, 1999.

Harris RA, O'Carroll DC, Laughlin SB. Contrast gain reduction in fly motion adaptation. Neuron 28: 595-606, 2000.

Hausen K, Wolburg-Buchholz W, Ribi WA. The synaptic organization of visual interneurons in the lobula complex of flies. A light and electron microscopical study using silver-intensified cobalt-impregnations. Cell Tissue Res 208: 371-387, 1980.

Hengstenberg R. Spike responses of "non-spiking" visual interneurone. $\mathrm{Na}$ ture 270: 338-340, 1977.

Hengstenberg R, Hausen K, Hengstenberg B. The number and structure of giant vertical cells (VS) in the lobula plate of the blowfly Calliphora erythrocephala. J Comp Physiol A Sens Neural Behav Physiol 149: 163-177, 1982.

Ivanov AI, Calabrese RL. Modulation of spike-mediated synaptic transmission by presynaptic background $\mathrm{Ca}^{2+}$ in leech heart interneurons. J Neurosci 23: 1206-1218, 2003.

Ivanov AI, Calabrese RI. Spike-mediated and graded inhibitory synaptic transmission between leech interneurons: evidence for shared release sites. J Neurophysiol 96: 236-251, 2006.

Juusola M, Uusitalo RO, Weckstrom M. Transfer of graded potentials at the photoreceptor-interneuron synapse. J Gen Physiol 105: 117-148, 1995.

Kalb J, Egelhaaf M, Kurtz R. Robust integration of motion information in the fly visual system revealed by single cell photoablation. J Neurosci 26: 7898-7906, 2006.

Karmeier K, Krapp HG, Egelhaaf M. Robustness of the tuning of fly visual interneurons to rotatory optic flow. J Neurophysiol 90: 1626-1634, 2003.

Krapp HG, Hengstenberg B, Hengstenberg R. Dendritic structure and receptive-field organization of optic flow processing interneurons in the fly. J Neurophysiol 79: 1902-1917, 1998.

Krapp HG, Hengstenberg R, Egelhaaf M. Binocular contributions to optic flow processing in the fly visual system. J Neurophysiol 85: 724-734, 2001.

Kretzberg J, Egelhaaf M, Warzecha AK. Membrane potential fluctuations determine the precision of spike timing and synchronous activity: a model study. J Comput Neurosci 10: 79-97, 2001.

Kurtz R, Warzecha AK, Egelhaaf M. Transfer of visual motion information via graded synapses operates linearly in the natural activity range. $J \mathrm{Neu}$ rosci 21: 6957-6966, 2001.

Laughlin SB. A simple coding procedure enhances a neuron's information capacity. Z Naturforsch [C]: 36: 910-912, 1981.

Luksch H, Khanbabaie R, Wessel R. Synaptic dynamics mediate sensitivity to motion independent of stimulus details. Nat Neurosci 7: 380-388, 2004.

Maddess T, Laughlin SB. Adaptation of the motion-sensitive neuron H1 is generated locally and governed by contrast frequency. Proc $R$ Soc Lond $B$ Biol Sci 228: 251-275, 1985.

Marder E. Extending influence. Nature 441: 702-703, 2006.

Meinrenken CJ, Borst JG, Sakman B. Calcium secretion coupling at calyx of Held governed by nonuniform channel-vesicle topography. J Neurosci 22: 1648-1667, 2002.

Redman S. Quantal analysis of synaptic potentials in neurons of the central nervous system. Physiol Rev 70: 165-198, 1990.

Rose GJ, Fortune ES. Frequency-dependent PSP depression contributes to low-pass temporal filtering in Eigenmannia. J Neurosci 19: 7629-7639, 1999.

Shu Y, Hasenstaub A, Duque A, McCormick DA. Modulation of intracortical synaptic potentials by presynaptic somatic membrane potential. Nature 441: 761-765, 2006

Simmons PJ. Transmission mediated with and without spikes at connections between large second-order neurones of locust ocelli. J Comp Physiol 147: 401-414, 1982.

Simmons PJ. The performance of synapses that convey discrete graded potentials in an insect visual pathway. J Neurosci 19: 10584-10594, 1999.

Simmons PJ. Signal processing in a simple visual system: the locust ocellar system and its synapses. Microsc Res Tech 56: 270-280, 2002. 
Simmons PJ, de Ruyter van Stevenick R. Reliability of signal transfer at a tonically transmitting, graded potential synapse of the locust ocellar pathway. J Neurosci 25: 7529-7537, 2005.

Sterling P, Matthews G. Structure and function of ribbon synapses. Trends Neurosci 28: 20-29, 2005.

von Gersdorff H, Matthews G. Electrophysiology of synaptic vesicle cycling. Annu Rev Physiol 61: 725-752, 1999.

Wadiche JI, Jahr CE. Multivesicular release at climbing fiber-Purkinje cell synapses. Neuron 32: 301-313, 2001.
Warzecha AK, Egelhaaf M. Neural encoding of visual motion in real-time. In: Vision: Computational, Neural, and Ecological Constraints, edited by Zanker JM, Zeil J. Berlin: Springer-Verlag, 2001, p. 239-277.

Warzecha AK, Kretzberg J, Egelhaaf M. Temporal precision of the encoding of motion information by visual interneurons. Curr Biol 26: 359-368, 1998.

Warzecha AK, Kurtz R, Egelhaaf M. Synaptic transfer of dynamic motion information between identified neurons in the visual system of the blowfly. Neuroscience 119: 1103-1112, 2003. 